\title{
Les sculptures funéraires de Cyrénaïque sur le marché de l'art
}

\author{
By Morgan Belzic"
}

\begin{abstract}
In the course of my research on Cyrenaican funerary sculptures, such as the remarkable 'Mourning Women' and 'Funerary Divinities' and the distinctive local funerary portraits, I realised to my dismay that a large part of this archaeological material has been or is currently on sale on the international art market. The number of sales of these sculptures on the art market demonstrates the extent of looting over the past twenty years in the Greek necropoleis of Libya. These sales show in particular that the degree of tomb destruction has increased exponentially during the past ten years. This preliminary discussion has three main objectives: 1) to alert and to inform the world about this destruction in order to help end the looting; 2) to describe the operational modes of the illicit trade in antiquities on the art market; and 3) to study and document these sculptures, which are important evidence for understanding the culture and history of ancient Cyrenaica.
\end{abstract}

أثناء قيامي بأبحاثي حول منحوثات برقة الجنائزية مثل "النساء في الحداد" و" آلهة

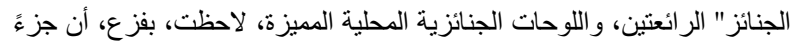

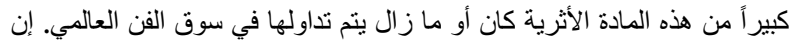

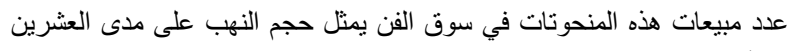

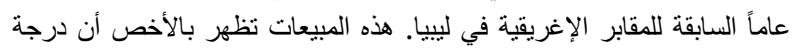

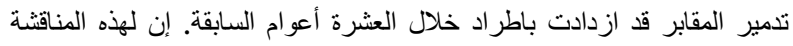

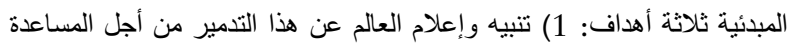

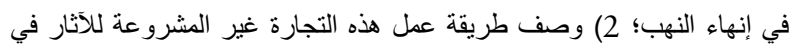

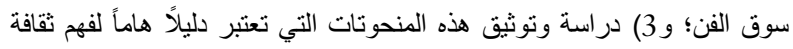

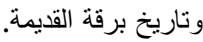

Cyrène et sa sphère d'influence sont remarquables à deux titres en ce qui concerne l'étude de l'architecture et de la plastique grecque depuis l'époque archaïque jusqu'à l'époque impériale : d'une part par la capacité des Cyrénéens à suivre les courants dominants par le jeu des importations ou de créations inspirées, d'autre part avec la mise au point de formes sculpturales proprement locales créant des registres d'expression novateurs où se mêlent adaptations stylistiques et innovations formelles. En outre, l'emploi massif de marbres importés, en parallèle aux matériaux locaux, distingue la Cyrénaïque de la plupart des autres régions colonisées par les Hellènes. Il est pourtant très difficile, en l'absence de contexte archéologique ou de

\footnotetext{
* Ecole Pratique des Hautes Etudes et Centre de Recherche sur la Libye Antique, Paris.
}

documents fiables, de pouvoir attribuer aux ateliers cyrénéens une sculpture provenant du marché de l'art. Les pilleurs et les vendeurs impliqués dans les vols dont sont victimes les sites archéologiques en Libye profitent de notre incapacité à distinguer avec une précision d'ordre juridique une statue d'Aphrodite sculptée à Ptolémaïs d'une autre créée à Milet, du fait de la mobilité des schémas, des modèles, des matériaux, des œuvres et des sculpteurs eux-mêmes depuis l'époque archaïque. Affirmer que tel kouros est de style naxien n'en fait pas une œuvre provenant de l'île de Dionysos. Néanmoins, il est des catégories suffisamment originales, de par leur forme et leur style, pour permettre d'en identifier la région d'origine. À ce titre, la Cyrénaïque est un modèle du genre, surtout en ce qui concerne les sculptures funéraires.

En effet, la part d'originalité des ateliers cyrénéens a essentiellement trouvé sa voie parmi les morts. Les vastes et monumentales nécropoles de Cyrénaïque, en premier lieu celles de Cyrène et de sa chôra, furent en effet des espaces de grande créativité où l'hétérogénéité des architectures se heurte, curieusement, à une relative homogénéité des marqueurs funéraires, particulièrement distincts des autres productions méditerranéennes. Ils sont malheureusement, du fait de leur vulnérabilité, de leur particularité ou de leurs qualités plastiques, victimes d'un important trafic.

\section{Divinités et portraits funéraires de Cyrénaïque}

Deux traditions de marqueurs funéraires ont dominé les nécropoles de la région. La première, la plus remarquable et originale, fut celle des sculptures dites divinités funéraires ${ }^{1}$ provenant essentiellement de Cyrène et de son port devenu Apollonia, ainsi que de Barca et de son port septentrional devenu Ptolémaïs. Rondes-bosses ou haut-reliefs féminins, majoritairement en marbre, elles sont abrégées en partie inférieure, déclinées en bustes ou demi-statues. De dimensions très variées, de quelques centimètres à deux mètres de hauteur, elles peuvent être réparties dans des proportions presque identiques en deux groupes, en fonction de la présence ou non d'un visage, entre représentations aprosopes et figurées (Ferri 1929). Si l'aprosopie semble avoir été la forme primaire de cette divinité, dès la fin du 
VIe s. (Fabbricotti 1996), elle perdure à côté des œuvres prosopiques depuis le Ve s. jusqu'à la fin de la production, que l'on peut situer au courant du Ier s. av. J.-C. Amplement étudiées par l'érudit L. Beschi, elles se décomposent en de nombreuses variétés déclinant des schémas que l'auteur divise en quatre groupes et vingt types nommés $\mathrm{A}$ à $\mathrm{V}$ (Beschi 1972, 335-336) selon des modalités qui doivent être réadaptées actuellement. Placées généralement au sommet des sarcophages ou en façade des monuments funéraires et ce quelle que soit leur forme architecturale, elles furent accompagnées entre le milieu du IVe siècle et le IIe s. av. J.-C. de bases inscrites au nom du défunt, le plus souvent masculin, attestant qu'il ne s'agit nullement d'un substitut du mort mais d'une représentation générique vraisemblablement divine. Ses rares attributs permettent de l'associer à l'iconographie de Perséphone (Beschi 1972, 335-336) bien qu'elle ne reçut probablement jamais de nom (Reynolds et Thorn 2005). Un anonymat qui résonne comme un écho à l'aprosopie.

La deuxième tradition, qui lui succède, directement ou plus certainement après une période de latence pour les ateliers, fut celle, d'origine italique, des portraits funéraires ${ }^{2}$. Hauts ou bas-reliefs féminins et masculins en position principalement frontale, arborant une portion variable du cou ou $\mathrm{du}$ thorax, ils sont généralement compris dans un format rectangulaire ou trapézoïdal et munis deux bandes de marbres relativement lisses dépassant de la plaque de fond de part et d'autre du cou. Ils étaient destinés généralement à être intégrés dans des niches creusées en façade de monuments funéraires souvent remployés (Di Valerio et al. 2005). Ils sont géographiquement plus diffus mais moins étendus chronologiquement. La principale étude qui en fut faite est intégrée au Catalogue of Cyrenaican Portrait Sculpture d'E. Rosenbaum (1960, $\left.\mathrm{n}^{\circ} 185-283\right)$ et ils ont été fréquemment réexaminés depuis (Oldjira et Walker 2016). Aucun n'a été découvert en contexte. La chronologie relative établie par Rosenbaum marque la prédominance des traits et coiffures des Ier et IIe s. de notre ère. De trop rares exemplaires pourraient être antérieurs, tandis que la production paraît s'éteindre à l'aube du IVe s.

Près de 190 divinités funéraires et davantage de portraits sont actuellement publiés ou mentionnés ${ }^{3}$. S'il existe une troisième tradition considérable, liée aux deux autres, celle des "stèles libyques " anthropomorphes trouvées pour l'essentiel autour de certains établissements de la chôra (Bacchielli 1987), elle ne semble pas avoir attiré le regard des collectionneurs anciens et modernes.
E. Rosenbaum, L. Beschi et M. Bonnano ont tour à tour établi les listes des divinités et portraits en et hors de Libye. Si les divinités funéraires ont été peu étudiées, et qu'il aura fallu plus d'un siècle pour prendre conscience de leur originalité, quelques-unes ont été exportées de Libye assez anciennement. Une divinité est au Louvre depuis 1851 (Beschi 1972, n'105; Zagdoun 2004, n'17), une autre fut acquise avant 1911 à Tripoli par le musée archéologique d'Istanbul (Beschi 1972, n89). Deux têtes prosopiques identifiées par L. Beschi comme des divinités funéraires sont au British Museum : la première provient des fouilles de Smith et Porcher en 1860-1861, la seconde fut acquise sur le marché de l'art londonien en 1936 (Beschi 1972, $\mathrm{n}^{\circ} 143$ et 145). Les portraits, sans doute plus nombreux originellement et de dimensions modestes, sont plus mobiles. Deux sont entrés dans les collections du Louvre (Zagdoun 2004, n²5-26), six du British Museum (Rosenbaum 1960, 137), un se trouve au Museo Nazionale Romano (Bacchielli 1977a), un autre au Musée d'Alexandrie (Bacchielli 1977b), six à Malte (Bonanno 1976), cinq en Crète et un à Athènes (Beschi 1976), un à Smyrne (Rosenbaum 1960, app. II n²) et un dernier au musée Rodin à Paris (Beschi 1976, 393), par les aléas du collectionnisme ancien, dès avant la conquête italienne de 1912. L'essentiel des objets conservés se trouvent donc actuellement aux musées de Cyrène, Apollonia, Ptolémaïs, Tripoli et Derna. De nombreuses divinités funéraires sont conservées en plein air, que ce soit autour des musées et des bureaux du Département des Antiquités ou à proximité du Caesareum de Cyrène où elles ont été entreposées afin de les soustraire au pillage, tandis que d'autres ont été signalées encore récemment in situ.

\section{Un constat dramatique}

Les recherches menées par les savants dans les années 50 et 60 permettent de dessiner un état des lieux très précis avant les conventions internationales des années 1970, ratifiées par la Libye, interdisant l'exportation des artéfacts archéologiques. Le contrôle étroit des archéologues italiens, britanniques et libyens sur les sites a grandement contribué au faible nombre d'œuvres exporté entre 1912 et 1970.

Nonobstant cette législation naissante, les années 1970 devaient pourtant initier une véritable rupture. On assiste d'une part à l'urbanisation galopante sur les périphéries des sites archéologiques, à commencer par Cyrène suite au plan d'aménagement du village de New Shahat sur la nécropole Sud en 1968, et d'autre part au désintérêt manifeste du nouveau 
gouvernement de Kadhafi installé en 1969. Cette réurbanisation progressive a empiété à Cyrène, comme à Apollonia, Ptolémaïs et Barca, sur l'emplacement des anciens cimetières que rend vulnérable leur position extra-urbaine, tandis que Benghazi connaissait un étalement urbain ayant les mêmes conséquences au détriment des nécropoles d'Euhespérides et de Bérénikè. Il en a résulté une multiplication des découvertes accidentelles, tantôt signalées ou repérées par les archéologues et donnant lieu à des fouilles d'urgence (Frigerio 1997 ; Thorn 2005, 116), tantôt probablement gardées secrètes. Dans le même temps, la pression démographique provoquait la création de nouvelles routes et chemins de desserte rendant facilement accessibles des portions complètes du territoire jusque-là éloignées et nécessitant une relative expertise et une certaine logistique pour exploiter les tombes. De fait, à Cyrène, le constat que dressait dès le début des années 1980 J. C. Thorn était alarmant. Celui dressé depuis le début des années 2000 par les membres de l'Université de Chieti l'est davantage (Menozzi 2015). Le nombre de pillages signalés est en constante augmentation et les destructions se font plus nombreuses, accompagnés de vandalisme et de pollution. La crise politique ouverte depuis la révolution de 2011 a accéléré le phénomène par la dérégulation des autorisations de construction et accru la difficulté, malgré leur énergie, des membres du Département des Antiquités pour protéger toute la région.

Il est impossible d'estimer les pertes dans leur ensemble. Si les tombes de Cyrénaïque sont généralement pauvres en matériaux précieux, elles peuvent contenir de nombreux verres, albâtres, terres cuites et céramiques plus ou moins luxueuses, dont les formes sont moins caractérisées localement, quand ce ne sont pas des importations. Une fois ces objets exportés sur le marché de l'art, il devient presque impossible d'en reconnaître l'origine. Ce n'est heureusement pas le cas des divinités et portraits funéraires de Cyrénaïque. Quelques critères simples permettent de facilement les identifier et les classer par groupes et par période au sein d'un vaste ensemble documenté. Doublement locales, de par leur iconographie et leur style, elles permettent de constater objectivement l'ampleur des fouilles illégales.

Notre étude a débuté en 2014, dans l'optique de compléter et d'actualiser le catalogue et les études sur les divinités funéraires (Belzic 2015a). En l'espace de presque cinquante ans, le Département des Antiquités de Shahat et Susa, de même que les archéologues des missions étrangères, ont en effet accumulé les documents sur près d'une centaine de pièces inédites, tandis que diverses saisies judiciaires sur le marché de l'art avaient déjà intercepté huit exemplaires. Très vite, de simples recherches sur internet ont permis de déceler une vingtaine de sculptures inédites actuellement ou récemment vendues par diverses sociétés en Europe et aux États Unis. Il est apparu que les divinités funéraires étaient vendues de manière concomitante avec les portraits funéraires. En novembre 2015 , la saisie en Égypte de six sculpture cyrénéennes comprenant trois portraits et deux divinités funéraires (Belzic 2015b) attestait ce qui était déjà soupçonné : les sculptures sont exportées depuis les nécropoles cyrénéennes par lots après regroupement avant d'être revendues sur le marché de l'art. En 2015 et 2016, l'extension de cette recherche aux deux catégories de matériel, en portant attention à la fois aux ventes publiques, aux galeries spécialisées et aux saisies, a entraîné le recensement de plus d'une centaine d'exemplaires, 42 divinités et 63 portraits funéraires actuellement ${ }^{4}$, accompagnés parfois de sculptures moins caractérisées mais de facture probablement cyrénéenne.

L'intérêt de cette prospection dans les archives des sociétés de vente est double. Elle permet de mieux comprendre et de lutter contre le trafic des biens culturels en documentant des œuvres au moment de leur disparition, qui sera parfois définitive, tout en contribuant, par les éclairages qu'apporte chacun de ces nombreux exemplaires inédits, aux études historiques et artistiques de l'ancienne Cyrénaïque. Le constat actuel est pourtant douloureux, puisque portraits et divinités pris ensemble, les œuvres du marché de l'art composent entre 15 et $20 \%$ des sculptures funéraires actuellement connues. Même avec beaucoup d'optimisme, il serait difficile de croire que nous ayons été capable de repérer ne serait-ce que la moitié des sculptures écoulées. Une part substantielle des deux corpus, avec les connaissances que nous pourrions en retirer, est donc en train de disparaître à tout jamais.

\section{Les saisies : les exemples de Londres, Genève et Damiette}

Les résultats de cette recherche ne sont en aucun cas issus d'un travail parfaitement solitaire. Une partie des œuvres nous ont été communiquées par de nombreux acteurs académiques ou institutionnels, en particulier en ce qui concerne les saisies ${ }^{5}$. Quatre divinités funéraires ont été saisies en France en 2012, cinq en Suisse depuis 2013, une au Royaume-Uni en 2013, deux en Égypte en 2015, accompagnées de trois portraits funéraires, soit un total de quinze sculptures portées à notre 
connaissance. Une dernière, plus récemment, en France, a fait l'objet d'un retrait pour vérification. Seule l'instruction judiciaire de la statue saisie à Londres est terminée et rendue publique : elle seule peut donc faire l'objet d'une publication préliminaire, permettant de prendre conscience de l'importance de chaque objet (Figures 1-3) ${ }^{6}$.

Exportée par un marchand d'art dubaïote tristement connu des autorités britanniques, Hassan Fazeli, la divinité funéraire nº́c.D23 (fig. 85) était notifiée comme "décor en marbre " ayant "plus d'un siècle ", originaire de Turquie, d'une valeur de $110000 £$, provenant de la « collection personnelle de M. Fazeli depuis $1977{ }^{7}$. Arrivée sur le sol britannique en 2011, elle a été restaurée en 2012 par Colin Bowles à la demande des vendeurs, $\mathrm{M}$. Al Qassas et M. Yaghi. Ce dernier aurait connu la statue à Dubaï cinq ans auparavant. L'œuvre a été saisie lorsqu'un employé du H.M.R.C. s'est inquiété du prix dérisoire de cette sculpture ridiculement intitulée "grecque du XVIIe siècle ». Elle a fait l'objet d'un procès médiatisé - une première ! - entre mars et

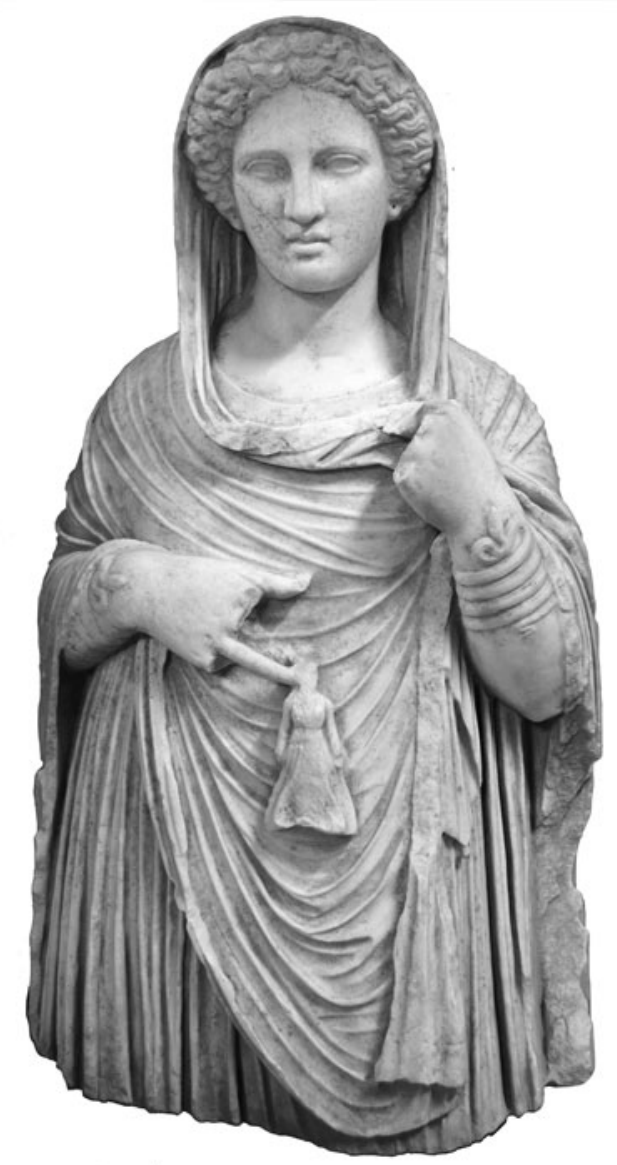

Figure 1. Divinité funéraire $n^{\circ}$ réc.D23 saisie à Londres. Photographie : British Museum.

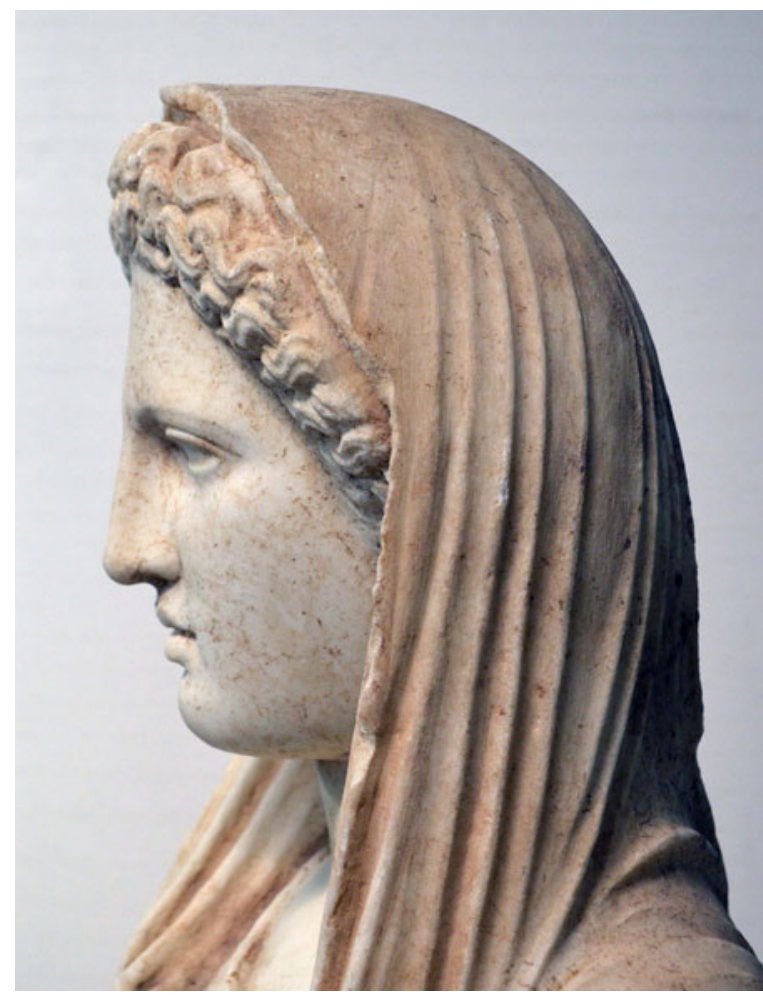

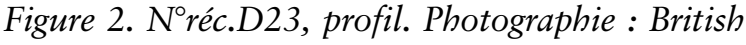
Museum.

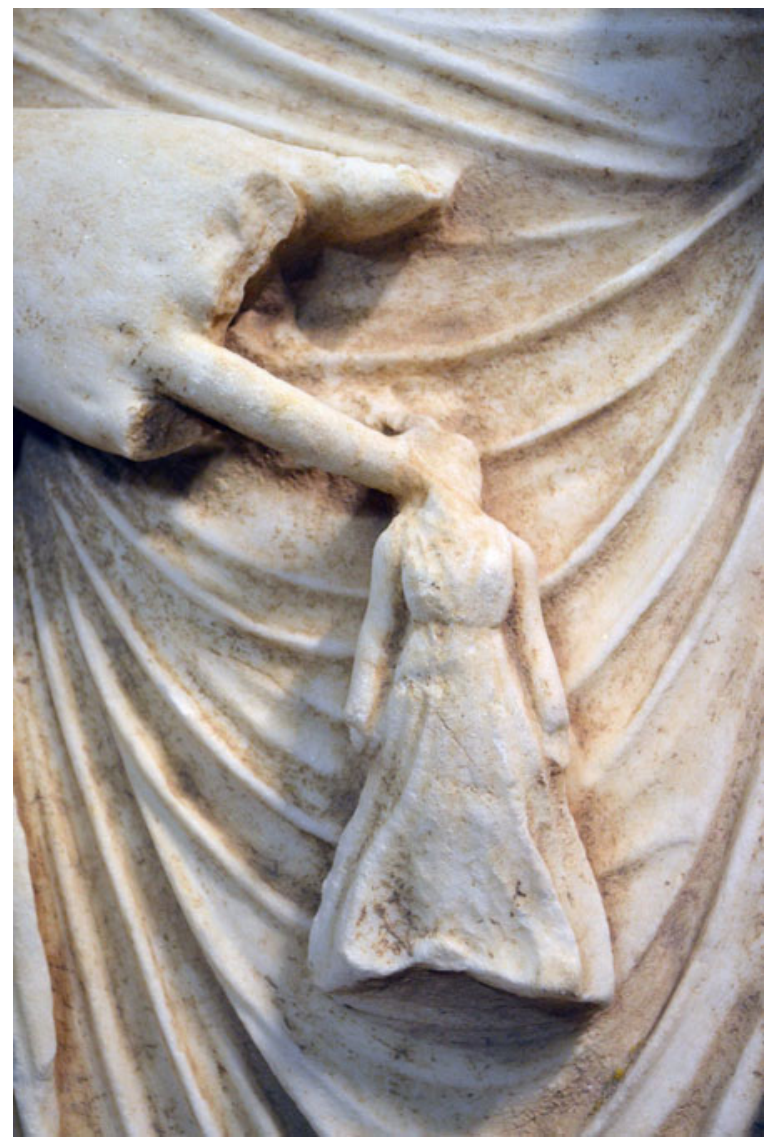

Figure 3. $N^{\circ}$ réc.D23, attribut. Photographie : British Museum. 
septembre 2015. L'œuvre a été estimée pour une somme bien plus élevée par les témoins lors du procès. La statue londonienne appartient au schéma le plus commun du "groupe canonique ", (type Beschi O), disposant d'un chiton cannelé et d'un himation dont les plis linéaires continus, similaire à la n¹18 par exemple, sont caractéristiques d'une mode interne à cette production à l'époque hellénistique. La proximité de cette formation des plis avec une sculpture de la tombe $S 4$ autorise à supposer qu'elle appartient à une production du IIe siècle avant J.-C (Beschi 1972, 303 ; Cherstich 2006, 110). Le doux visage classicisant ovale, proche des n ${ }^{\circ} 29$ et 141 , dispose d'une coiffure relativement volumineuse qui est à rapprocher de la tête ${ }^{\circ}$ réc. D28 déposée au Metropolitan Museum de New York (Llewellyn-Jones 2003, 318, fig.173, sans identification). Cette comparaison est d'un intérêt particulier. En effet, cette tête en dépôt, qui proviendrait d'une collection belge, est présentée au musée comme funéraire mais sans indication sur sa provenance pourtant évidente puisque ses traits sont partiellement couverts d'un voile, faisant disparaître la partie droite du visage, ce qui constitue un phénomène interne propre aux divinités funéraires de Cyrénaïque connu par trois autres sculptures (Beschi 1972, n`57, 1158 et 159) et qui n’a pas trouvé de parallèle ailleurs. Dater la statue londonienne permet donc de situer plus précisément une remarquable tentative des sculpteurs cyrénéens de créer un intermédiaire visuel entre les divinités avec et sans visage au cœur de l'époque hellénistique, qui marque probablement une des acmés de la production.

Malgré le nettoyage profond que la demi-statue londonienne a subi, la surface du marbre laisse encore entrevoir les traces orangées des dépôts de terre souvent marqués en Cyrénaïque par une teinte ferrugineuse (alors nommée terra rossa) mais qui peut revêtir selon les lieux, à Cyrène en particulier, un aspect ocre ou jaunâtre. Comme il a déjà été remarqué (Beschi 1972, 313-314), les traces d'outils sont nombreuses par endroit, que ce soit au dos, par absence de nécessité de peaufiner une zone qui demeure le plus souvent peu visible, ou sur les plis du drapé, soit par souci de rapidité d'exécution soit par envie de donner au vêtement une texture proche du textile; il n'est que de remarquer à ce propos le contraste avec le soyeux des chairs. Le dos est travaillé comme rarement, bien que de manière plus graphique, d'un jeu de longues lignes parallèles savamment entrecroisées.

Enfin, sans détailler ici tous les apports de cette sculpture, elle renouvelle profondément l'étude des divinités funéraires en raison de son attribut suspendu au bout de la main droite (Figure 3), une figurine féminine, les bras le long du corps, vêtue d'une tunique en corolle qui n'est pas sans rappeler l'image d'Hécate, gardienne des portes des enfers et témoin essentiel du rapt de Perséphone. Inconnu jusqu'ici, ce type d'attribut trouve une correspondance dans la demi-statue $\mathrm{n}^{\circ}$ réc.D24, véritable chef-d'œuvre volé dans la nécropole de Cyrène et connu depuis 2013 par une vidéo de piètre qualité ayant circulé sur internet. Une photographie plus récente atteste la proximité des deux sculptures, bien que le drapé plastique et animé de la divinité filmée relève d'une plus grande proximité avec les sculptures du IIIe s. et puisse être antérieur, s'il ne s'agit pas là simplement d'une différence d'atelier. Son attribut se distingue de la statuette londonienne par sa nudité et sa position hiératique: elle rappelle davantage les statuettes d'Isis-Aphrodite d'époque hellénistique ou les « poupées » en terre cuite que l'on trouve parfois dans le mobilier funéraire, à Cyrène comme ailleurs ${ }^{8}$. Poupées ou statuettes de déesse portées en talisman, il est difficile de se prononcer. Il est bien possible qu'il y ait, comme souvent dans cet ensemble sculptural et au-delà des frappantes limites de nos connaissances, une troublante opacité volontairement et soigneusement entretenue. Si cette sculpture avait disparu, nous serions passés à côté d'éléments-clés pour l'établissement de critères chronologiques et l'analyse iconographique.

Une saisie récente, à Genève, dont les photographies sont parues dans la presse ${ }^{9}$, met en évidence la présence d'un lot complet provenant sans doute d'une même sépulture, probablement à Cyrène ou Apollonia. Trois divinités funéraires, d'époque hellénistique elles aussi, montrent une similarité de style de drapés et de coiffures, revêtant la même patine ocre (réc.D30-32). L'une de ces œuvres (réc. D32) dispose d'un visage aprosope dont l'emplacement des traits est voilé. Cette image, liant aprosopie et masque du voile, déjà partiellement connue par un exemplaire inédit du Musée de Cyrène (FrontisiDucroux 2008), se double ici de la position inédite de la main portée au visage qui correspond à un geste de deuil effectué, en particulier, par l'un des personnages du célèbre Sarcophage des Pleureuses de Sidon au Musée Archéologique d'Istanbul.

Enfin, la saisie opérée en Égypte est intéressante en ce qu'elle n'a pas été faite, contrairement aux autres, lors de l'arrivée des sculptures sur le marché officiel mais au moment de leur départ (Figures 4-5). Une fouille de la police portuaire en novembre 2015 à Damiette ciblait un conteneur à destination de Bangkok, transportant, à la grande 


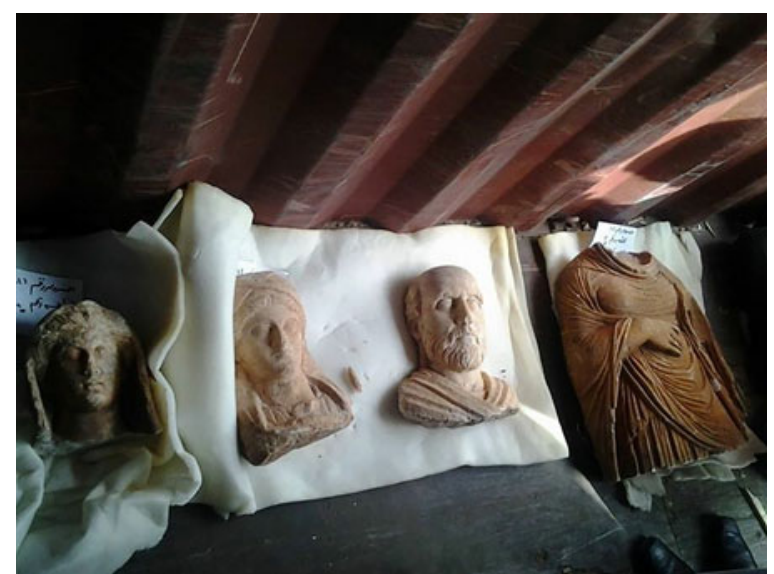

Figure 4. Divinité funéraire $n^{\circ}$ réc.D25 saisie à Damiette. Photographie : Autorités égyptiennes.

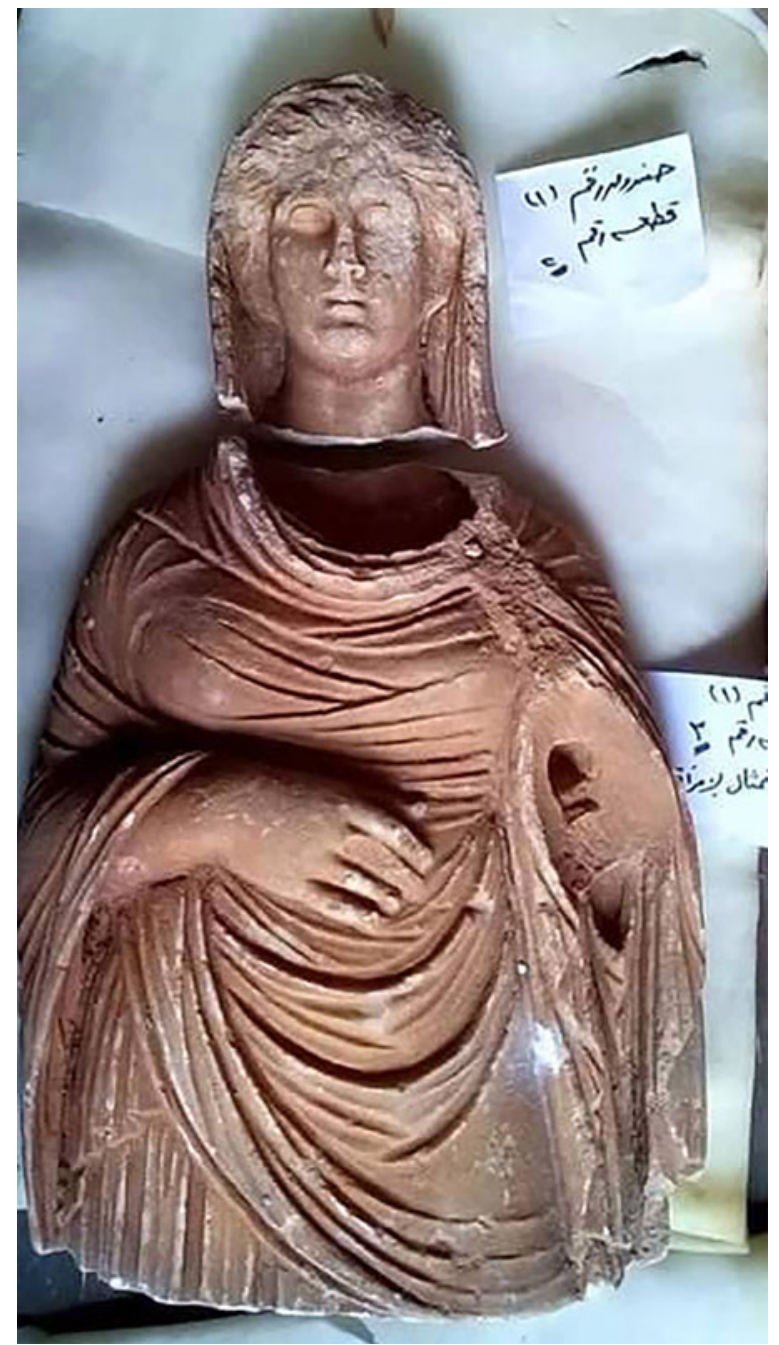

Figure 5. Intérieur du conteneur 8/861405 à destination Bangkok saisi à Damiette, avec les $n^{\circ}$ réc.D25, P5 et P6. Photographie: Autorités égyptiennes. surprise des fonctionnaires, plus d'un millier d'artefacts pharaoniques immédiatement saisis. Prévenu par hasard par un membre de la conservation du site de Memphis, nous avons pu reconnaître en leur sein un ensemble de cinq sculptures en marbre de Cyrénaïque (Belzic 2015b) : une divinité complète, bien que la tête ait été fractionnée suite au pillage ( $n^{\circ}$ réc.D25, fig. 5), une tête voilée très certainement de la même catégorie ( $n^{\circ}$ réc.D26) et trois portraits funéraires d'époque impériale (nréc.P5-7). L'ensemble, fortement imprégné de terra rossa, est issu de pillages récents à n'en pas douter, probablement à Cyrène. Une autre statuette féminine en marbre pourrait, étant donné le contexte, provenir de Cyrénaïque. Elle appartient cependant à un type plus générique. Quoi qu'il en soit, cet ensemble permet d'établir que les sculptures funéraires et sans doute votives sont regroupées par les trafiquants en Libye avant d'être transportées pour leur exportation via l'Égypte, essentiellement, depuis la crise de 2011. Il est possible que les objets soient dirigés vers des intermédiaires situés au Moyen-Orient avant d'être revendus par une série de galeries et de maisons de ventes, conservant parfois la formation des lots antérieurs.

\section{Galeries : les exemples de Tel Aviv et Barcelone}

Une observation essentielle explique le grand nombre d'objets relevés lors des recherches dans les archives numériques et papiers des sociétés de vente : il est très rare qu'une divinité ou un portrait soit vendu seul ${ }^{10}$. La plupart du temps, les galeries ou maisons sont à l'origine de ventes multiples, au sein d'un même catalogue ou écoulées de saisons en saisons.

À ce titre, deux galeries peuvent être citées comme exemplaires de cette situation. La première est la société Archaeological Center Ltd à Tel Aviv, qui a vendu deux portraits funéraires le 17 avril 2006 (réc.P50-51), deux autres le 6 octobre 2009 (réc.P10 et P49), un cinquième le 1er avril 2009 (réc.P48), deux portraits et deux divinités conjointement le 7 avril 2015 (réc.P45-46 et D40-41) et un dernier portrait le 26 avril 2016 (réc.P47). Cela compose un total de dix sculptures à notre connaissance, qui pourraient n'être que le sommet de l'iceberg au vu du nombre impressionnant de céramiques, monnaies et verres mis en vente par le propriétaire, Robert Deutsch, tristement connu pour avoir poursuivi avec succès l'Israel Antiquities Authority (IAA) après avoir été acquitté en 2012 d'accusations de fraudes et de trafic illégal. Comment expliquer 
qu'un même point de vente puisse en l'espace de dix ans proposer dix sculptures, rarissimes à l'étranger, de même provenance libyenne?

La même question se pose avec la galerie J. Bagot à Barcelone. De constitution récente, cette société se distingue pourtant par la grande qualité et l'abondance des pièces archéologiques mises en vente. Entre 2014 et 2016, Jaume Bagot a vendu quatre corps de divinités funéraires, trois têtes apparentées et un portrait funéraire de Cyrénaïque, chaque pièce provenant selon lui de collections différentes. Au fur et à mesure, les identifications des divinités se font plus précises: il s'agit de Perséphone ou de Déméter, elles sont conçues pour le domaine funéraire, on cite directement Cyrène et la Libye ${ }^{11}$. Plusieurs notices renvoient même directement à Luigi Beschi. Une divinité funéraire acéphale (réc. D3), de type Beschi P, vendue en 2014, est dite provenir d'une "collection privée européenne » et être passée en vente en 2007 chez Christie's, ce que nous n'avons pas pu vérifier. Une seconde (réc.D4), de type Beschi I, mise en vente en même temps, proviendrait d'une " collection privée, Asie, depuis les années 1980 ». Une troisième (réc.D5), proposée en septembre 2015, proviendrait à nouveau d'une « collection particulière européenne ». Une quatrième (réc.D6), repérée en janvier 2016 et présentée à la Brussel Art Fair (Brafa) au printemps, proviendrait enfin d'une «collection S.G., Belgique » et est parfaitement identifiée comme cyrénéenne. Elle est de nouveau mise en vente en France quelques mois plus tard sous une autre appellation et sans mention de sa prétendue collection d'origine. Dans le même temps, J. Bagot mettait en vente trois têtes féminines voilées dont les caractéristiques renvoient, avec un faisceau de doute minimal, à la même catégorie (réc.D12-14). Deux d'entre elles ornent même la couverture des catalogues édités par J. Bagot pour les Brafa de 2015 et 2016 et proviendraient là encore de collections européennes ou asiatiques, indications d'une rare précision géographique, on en conviendra. Enfin, un portrait funéraire (réc.P12), proche en particulier du n ${ }^{\circ} 269$ (Rosenbaum 1960, 120), de teinte rougeâtre, serait issu d'une " collection privée F.T., Asie, acquise dans les années 1960 ». La notice explique que "sûrement, il s'agit d'un portrait funéraire, destiné à être placé dans la tombe (...). Il est possible de penser qu'il y avait une inscription faisant référence à son nom », et que le style est " classique » mais reçoit des " influences orientales, ce qui fait penser que cela pourrait être une œuvre produite dans cette zone ". Malgré le caractère léger de ces analyses stylistiques et géographiques, ces précisions démontrent que le vendeur connaît l'origine et la fonction de cette sculpture. C'est le constat général qui s'impose lorsque l'on songe à J. Bagot. C'est officiellement que sont vendues à Barcelone, en plein conflit libyen, des sculptures provenant de sites archéologiques de Cyrénaïque et probablement de Cyrène même, au vu de l'abondance et du style du matériel, site pourtant classé au Patrimoine Mondial de l'Humanité par l'UNESCO. Est-il possible que ces huit sculptures, jamais documentées, aient effectivement été exportées légalement hors de Libye avant 1970? Difficile de l'imaginer. Notons que J. Bagot n'est pas isolé dans la capitale catalane. Son voisin immédiat, la galerie F. Cervera, a aussi mis en vente en 2016 un portrait (réc.P9) et une tête de divinité funéraire (réc.D29). Car si les galeries J. Bagot et Archeological Center Ltd poussent le phénomène de concentration à l'extrême, il s'observe encore chez Goldberg à Los Angeles, Malter à Encino (CA), Malloy, Safani et Eisenberg à New York, Barakat à Londres, Biblical Artifacts et Baidun Shop à Jérusalem, Gorny \& Mosh à Munich.

\section{Maisons de vente : les exemples de Christie's et Bonhams}

L'analyse des catalogues de vente de Christie's à New York et Bonhams à Londres met en évidence des phénomènes similaires d'association lors des ventes.

Entre 2002 et 2012, Christie's a mis en vente à New York au moins cinq divinités et quatre portraits funéraires, certains lors des mêmes ventes. En juin et décembre 2007 sont mis en vente consécutivement un portrait funéraire et deux têtes vraisemblablement de divinités funéraires. Pourtant, ces trois sculptures proviendraient de trois collections différentes. Les 8 juin et 5 décembre 2012, Christie's a mis en vente trois sculptures identifiant clairement la collection privée, celle de Ralph W. Stephan dans les années 1960 puis de Catharina Baart-Biddle à Washington. La partie supérieure d'une divinité funéraire (réc. D16), dont la notice affirme directement l'origine cyrénéenne et renvoie au chapitre de L. Beschi dans le catalogue The Western Greeks (Beschi 1996), était associée à deux portraits funéraires masculins. Le premier (réc.P1), similaire aux $\mathrm{n}^{\circ} 192$ et 194 (Rosenbaum 1960,103), est complet et a été revendu le 1er décembre suivant par Ira et Larry Goldberg Coins and Collectibles. Le second, dont la partie inférieure est manquante, est cependant clairement identifié : «For related portraits from Cyrene, with similar flanges framing the neck, see nos. 271 and 273 in Walker and Bierbrier, Ancient Faces, Mummy Portraits from Roman Egypt " (Walker 
1997). Une courte recherche permet de comprendre que Catharina Baart-Biddle, artiste peintre décédée en 2005, fut l'épouse de R. W. Stephan en premières noces, Biddle en secondes noces. Au moins une autre sculpture antique est dite provenir de cette collection : une petite tête de déesse en marbre vendue par Christie's le 8 juin 2012 avec ces deux portraits, revendue par la Royal Athena Gallery à New York en 2013 lors de la même vente que la divinité funéraire réc.D $9^{12}$. Il s'agit là de l'unique collection dont nous ayons pu retrouver quelques traces, marquant une fois encore la concentration des objets au sein de lots de même nature, avec toutefois moins de doutes quant à la légalité de la vente.

À Londres, Bonhams a proposé huit portraits funéraires de Cyrénaïque et deux divinités funéraires entre 2006 et 2014. Parmi ces ventes, trois groupements peuvent être soulignés. Les 27 avril et 13 octobre 2006 sont vendus deux portraits funéraires dont la notice mentionne des collections initiales différentes (réc.P36 et P42). Le 26 octobre 2007, deux autres portraits mis en vente (réc.P16-17) sont clairement identifiés comme "Property of a private Scottish lady, inherited from her mother who acquired them in Libya pre 1960. They were discovered near Misurata in the early 1950's whilst a pipeline was being laid in the desert ". Néanmoins, ce serait une première bien étrange : Misrata se trouve en Tripolitaine et nul portrait cyrénéen n'y a été jusqu'ici découvert. Les 29 avril et 28 octobre 2009 sont mis en vente consécutivement un portrait féminin (réc. P15), ayant conservé la trace des agrafes permettant de la fixer dans une niche, puis une divinité funéraire de petites dimensions (réc.D33). Cette dernière, partageant un schéma déjà identifié $(\gamma)$, porte un nouveau type d'attribut, avec trois grenades reposant entre ses mains sur un pan de l'himation relevé afin de servir de panier. Symbole de vie et de mortalité, la grenade, associée au mythe de Perséphone (Apollodore, Bibliothèque, I, 5, 3.) avait été déjà observée sur l'exemplaire $n^{\circ} 6$ aujourd'hui disparu (Beschi 1972, 213). Le 2 octobre 2014 enfin, Bonhams mettait en vente encore deux portraits funéraires (réc.P40-41), provenant à nouveau, selon les notices, de deux collections différentes, l'une néerlandaise, l'autre britannique.

Il en va de même à Munich, Bruxelles et surtout Paris. Le célèbre hôtel des ventes Drouot a vu défiler, entre 2007 et 2015, un minimum de onze divinités et portraits funéraires de Cyrénaïque mis en vente par seulement trois sociétés, Pierre Bergé \& Associés, Delorme \& Collin du Boccage et Boisgirard \& Associés, témoignant des mêmes formes d'association et d'un autre phénomène plus complexe, celui du vaste système d'échange et de revente qui rendent le parcours de ces objets particulièrement chaotique.

\section{Échanges et reventes}

Avec ces marqueurs que sont les sculptures funéraires de Cyrénaïque, il est possible d'observer une part du fonctionnement du marché de l'art mondial, notamment en ce qui concerne la volatilité et la mobilité des objets, les liens et réseaux qui unissent entre elles certaines galeries et maisons de vente pourtant concurrentes. Un cas intéressant est offert par une supposée «Ex-Ebrahimoff collection » en "Suisse ", inconnue par ailleurs, qui apparaît dans les notices de deux divinités et deux portraits funéraires, pourtant vendus en 2009 et 2010 séparément par la Royal Athena Gallery d'Eisenberg à New York (réc. D15 et P19) ${ }^{13}$ et la société Pierre Bergé et Associés à Paris (réc.P29 et D37) ${ }^{14}$.

En réalité s'observe un échange fréquent entre les galeries et les maisons de vente, avec des sculptures issues de mêmes "collections » ou de mêmes ensembles vendus par différentes sociétés, parfois écoulés sur plusieurs mois, parfois en seulement quelques jours. De nombreuses sculptures passent ainsi d'une société à une autre, prenant ou perdant de la valeur d'une vente à l'autre, montrant un important système d'échange qui ne concerne bien entendu pas uniquement cette catégorie d'objets. Cela est davantage vrai pour les portraits ou têtes isolées :

- le portrait réc.P1 est vendu en juin 2012 par Christie's à New York et revendu au mois décembre suivant par Ira \&. Larry Goldberg à Los Angeles;

- le portrait réc.P4 est vendu en janvier 2009 par Pierre Bergé \& Associés à Paris, revendu en 2010 par Royal Athena Galerie à New York, stipulant qu'elle provient de la Galerie Serres à Paris;

- le portrait réc.P10 est vendu en octobre 2009 à Tel Aviv par Archeological Center Ltd, revendu en janvier 2016 par Baidun Shop à Jérusalem;

- le portrait réc.P25 est proposé à la vente à Paris le 27 Mai 2011 par Boisgirard \& Associés puis par Pierre Bergé \& Associés cinq jours plus tard le 1er juin 2011, finalement vendu en décembre suivant;

- le portrait réc.P28 est mis en vente à Paris par Pierre Bergé et associés en mai 2008 et revendu en septembre 2014 par G. H. Nachfolder à Munich;

- le portrait réc.P29 est vendu en juin 2010 par Pierre Bergé \& associés à Paris puis par Bonhams à Londres en avril 2015; 
- le portrait réc.P32 vendu à Munich par Gorny \& Mösch en décembre 2013 est revendu au RoyaumeUni par timelineauctions.com en février 2015;

- le portrait réc.P36 est vendu le 27 avril 2006 par Bonhams à Londres, revendu dans la même ville par Artemission Gallery en novembre 2010.

- la tête réc.D37 est mise en vente à Paris par Pierre Bergé et Associés en décembre 2009, par Bonhams à Londres en octobre 2011 puis par Gorny \& Mösch à Munich en juin 2013.

- la tête réc. D38 est vendue en 2010 à Paris par Pierre Bergé et Associés, se trouve aux États Unis en 2014 et est exposée par la galerie New-Yorkaise Safani à Bruxelles en 2016.

Aussi le parcours d'une œuvre est-il parfois difficile à retracer, même lorsque la notice est extrêmement détaillée. La divinité funéraire complète vendue par Christie's le 11 décembre 2009, ayant atteint la somme record de 422500 \$, en est l'illustration. Elle proviendrait de la "Collection Sarkis » en Suisse, dont nous n'avons pas de traces, excepté éventuellement une tête d'Hercule en marbre vendue par Christie's en 2012, la collection étant alors indiquée "Sarkis, Bayrut, 1941 "; il est à noter que cette tête est arrivée de Genève aux États Unis en 1985. Dans les années 1960, cette demi-statue est située par Christie's dans la collection Ishiguru ou Ishiguro, sur laquelle nous n'avons pas de données précises au Japon mais dont proviendrait une statuette de "dame de Bactriane ", ainsi que d'autres objets du Ghandhara et du Luristan, ou encore une "idole d'Amlash »; autant d'autres catégories d'objets victimes d'intenses pillages suite aux conflits en Afghanistan. Elle aurait été ensuite possédée par la société "Galerie Archaïque »au Japon, en cette même année 1985, dont nous n'avons guère pu ne serait-ce que prouver l'existence. Participant au type $S$ de Beschi, revêtant les traditionnels bracelets serpentiformes, la provenance et la fonction de cette demi-statue ne font aucun doute. Elle ouvre néanmoins la voie à l'identification d'une catégorie interne à la classe marquée par un vêtement et un visage plus enfantin. Il est désormais possible de la rapprocher d'une tête publiée par E. Paribeni (1959, n¹00) qu'il considérait alors comme votive. Cette approche est confirmée à présent par deux autres sculptures, l'une saisie à Paris (réc.D22), l'autre en vente à Londres en 2015 (réc.D34). La proximité de ces vêtements avec deux autres statues votives de jeunes filles de Cyrène (Paribeni 1959, n¹11-112) permet peut-être d'identifier un véritable travail d'atelier, actif à Cyrène probablement au IVe s. av. J.-C., marquant la polyvalence des sculpteurs.

Des irrégularités sont notables, à l'image du portrait nºréc.P32, désigné en 2013 à Munich comme provenant d'une collection allemande locale des années 1990, en 2015 au Royaume-Uni comme provenant d'une " importante collection du nord de Londres formée avant 1980 ». Enfin, soulignons quelques curiosités, comme en 2011 à Drouot, Paris. En plein début du conflit libyen, du 13 au 27 mai, trois maisons de ventes différentes s'échangent entre elles et vendent une série de cinq portraits grecs de Libye, sans mention des collections d'origine ou de la provenance des sculptures. Ce cas n'est malheureusement pas isolé, comme si des stocks étaient rapidement écoulés en divers points de vente. La question de la légalité de ces ventes se pose : comment ces rarissimes et très circonscrites productions pourraient-elles se retrouver en si grand nombre, au même moment, dans les mêmes ventes et vitrines ?

\section{Conclusions}

Nous devons avoir conscience que derrière chaque sculpture exportée, il y a un contexte archéologique définitivement détruit. La perte d'information est alors irrémédiable alors même que bien peu de contextes précis furent étudiés. Il y a là des lacunes de l'archéologie que le pillage massif dont sont victimes les nécropoles continue inlassablement d'augmenter, détruisant peu à peu le legs des anciens et nos chances d'en comprendre certains aspects.

Ces 105 sculptures funéraires provenant des nécropoles grecques de Cyrénaïque actuellement recensées nous autorisent à présupposer la présence d'un nombre absolument considérable de sculptures sur le marché noir, soit vendues de la main à la main, soit en attente de jours meilleurs dans les ports francs ou autres lieux de stockage de vendeurs avertis. Ce marché, dont on peut dire sans risque qu'il représente plusieurs millions d'euros, a connu une forte augmentation observable dès 2007, la plus sensible hausse couvrant les années 2015 et 2016. Des résultats peuvent être interprétés de plusieurs manières. Le premier facteur est à l'évidence la recrudescence des pillages et l'expansion urbaine ininterrompue et incontrôlée, amplifiés par la crise politique libyenne depuis la révolution de 2011. Le second est l'accessibilité des données, en particulier avec les ventes en ligne, toujours plus nombreuses au fur et à mesure des années. Enfin, le début même de cette recherche en 2014, amplifiée en 2016, explique à son tour l'accroissement des 
résultats : certaines œuvres disparaissent en effet sitôt vendues des sites et ne passent que quelques jours en ligne, nécessitant une veille permanente.

Il y a fort à parier que la majeure partie de ces sculptures a été sortie du territoire libyen illégalement puis revendue sous de fausses appellations ou de fausses mentions de collections originelles. Chaque vérification effectuée par les autorités a montré jusqu'ici à quel point le parcours de ces œuvres était une construction artificielle qui n'a rien à envier aux autres formes de trafic. Cette analyse est confortée par les diverses saisies effectuées depuis 2012. Il est intéressant à ce propos de constater que depuis les premières actions judiciaires en France, en Suisse et au Royaume-Uni, bien peu de sculptures cyrénéennes ont été vendues à Paris, Londres et New York. Aux grandes maisons de vente internationales succèdent donc une myriade de galeries plus éparpillées, essentiellement en Espagne et en Israël, ou prennent peut-être des directions différentes, vers le Moyen-Orient, la Russie et l'Asie Orientale. Le marché semble déjà se réorganiser.

Ce recensement est loin de pouvoir prétendre à l'exhaustivité, même relative. Pourtant, il permet d'élaborer quelques actions. Si l'on peut espérer restituer ponctuellement à la Libye quelques ouvres grâce à l'effort des acteurs impliqués dans la lutte contre le trafic des biens culturels, il faut aussi poursuivre deux objectifs prioritaires. Le premier est de juguler le trafic à la source, ce que tentent, sur place, les membres du Département des Antiquités de Shahat qui ont déjà, ces dernières années, efficacement incité les habitants à retourner près d'une centaine de sculptures dans les collections publiques. Le second est de documenter et d'étudier les sculptures repérées sur le marché de l'art, de manière à renouveler et compléter nos connaissances. En effet, chacune de ces sculptures participe d'une série tout en étant unique de par le style, le traitement, la gestuelle ou les attributs, ce qui permet d'identifier des phénomènes collectifs, que ce soit le contexte de création, le travail des ateliers, les modes de présentation ou les significations religieuses et sociales, tout en s'interrogeant sur le rôle et la place de l'individu et de son groupe, artistes et commanditaires, dans le choix des formules plastiques et iconographiques. Parmi les œuvres repérées, nombreuses sont celles qui apportent de nouveaux aspects à même de modifier, voire de bouleverser, les analyses qui avaient étaient faites précédemment par l'apparition de nouveaux schémas, attributs ou modes de représentation des drapés, ou par le complément de séries connues mais peu documentées. Aussi cette recherche doit-elle se poursuivre dans les années qui viennent afin que peu à peu nous puissions, à défaut de ne pouvoir réparer les dommages irréversibles causés par la destruction des contextes archéologiques, en atténuer quelque peu les effets.

\section{Notes}

1 Beschi 1972 ; Cassels 1955 ; Chamoux 1953, 193 s. ; Fabbricotti 1996 et 2002 ; Ferri 1929 ; Siciliano 2006.

2 Rosenbaum 1960, 13 ; Bacchielli 1977a ; Beschi 1976; Bonanno 1975-1976 ; Bonanno 1976 ; Bonanno Aravantinos 2007 ; Giovannini 2014, 67 ; Marini 2012 ; Oldjira and Walker 2016.

3 Nous connaissons actuellement plus de 300 divinités funéraires et 410 portraits, dont les catalogues complémentaires sont en cours de création.

4 Nous employons ici, de manière provisoire, la numérotation du récolement divisée entre les deux catégorie principales : $\mathrm{n}^{\circ}$ réc.D pour les divinités funéraires, réc.P pour les portraits funéraires.

5 Je remercie tout particulièrement Vincent Michel, Oliva Menozzi, Susan Kane, Ahmed Hossein et Ahmed Dakil Al Hasi pour leur aide.

6 La demi-statue est actuellement conservée au British Museum en attendant que les conditions de son retour soient optimales. Je remercie Peter Higgs pour l'accès à la statue et aux photographies.

7 John Zani, rapport "In the Westminster Court between H. M. Revenue \& Customs v Riad Issa Mohamad Al Qassas ", 1er septembre 2015.

8 Par exemple au Musée du Louvre la terre cuite MN 1012.

9 http://www.corriere.it/esteri/16_marzo_04/libia-rischioanche-decine-tesori-archeologici-stilata-red-list-850e8068e1e2-11e5-b31b-034bb632a08d.shtml consulté le 16/04/ 2016.

10 Nous ne pouvons ici insérer tous les liens hypertextes, trop nombreux et dont le format et le langage ne conviennent pas à un format d'article. Ils seront en revanche disponibles dans la publication définitive.

11 J. Bagot, Archaeology, Ancient Art, Brafa (Brussels Art Fair) 2015 et 2016.

12 Eisenberg, J. Royal Athena Gallery, Art of the Ancient World, vol. XXIV, 2013, p. 5.

13 Eisenberg, J. Royal Athena Gallery, Art of the Ancient World, XXI, 2010, p. 5.

14 Godeau, A., Chambre, F. Pierre Bergé \& associés, Archéologie, 5 décembre 2009, №64 p. 148 ; Idem, Pierre Bergé \& associés, Archéologie, jeudi 17 juin 2010, No305 p. 207. 


\section{References}

Bacchielli, L. 1977a. Un ritratto cireneo nel Museo Nazionale Romano e alcune osservazioni sui busti funerari per nicchia, Quaderni di Archeologia della Libia 9: 77-92.

Bacchielli, L. 1977b. Un ritratto cirenaico in gesso nel Museo Greco-Romano di Alessandria, Quaderni di Archeologia della Libia 9: 97-110.

Bacchielli, L. 1987. « La scultura Libya in Cirenaica et la variabilità delle risposte al contatto culturale greco-romano ", Quaderni di Archeologia della Libia 12; Cirene e i Libyi : 459 s.

Belzic, M. 2015a. Les divinités funéraires de Cyrénaïque, un état de la recherche, mémoire d'étude inédit, École pratique des Hautes Études, Paris.

Belzic, M. 2015b. Report: Cyrenaican Funerary Sculptures seized in Damietta, inédit, 2015.

Beschi, L. 1972. "Divinità funerarie Cirenaiche ", Annuario della scuola italiana di Atene e delle Missioni Italiane in Oriente 47-48, n.s. 21-22 (1969-70), 1972: 133-341.

Beschi, L. 1976. Un supplemento cretese ai ritratti funerari romani della Cirenaica. Quaderni di Archeologia della Libia 8: 385-397.

Beschi, L. 1996. La scultura nella Cirenaica greca. I Greci in Occidente. Catalogo della mostra Venezia, Palazzo Grassi, marzo - dicembre 1996, Venise-Milan, 1996: 437-442.

Bonanno, A. 1975-1976. Another funerary portrait from Cyrenaica in the British Museum. Libyan Studies 6: 27-30.

Bonanno, A. 1976. Cyrenaican funerary portraits in Malta. Journal of Roman Studies, 66: 39-44.

Bonanno Aravantinos, M. 2007. I ritratti di età romana della Cirenaica: lo stato degli studi. Considerazioni sui ritratti dal Tempio di Zeus Olimpio a Cirene. In Gasperini, L., Marengo, S.M. (eds), Cirene e la Cirenaica nell'Antichità. Atti del Convegno Internazionale di Studi, Roma-Frascati 18-21 dicembre 1996, Macerata: 87-104.

Cassels, J. 1955. "The Cemeteries of Cyrene ", Papers of the British School at Rome 23: 1-43.

Chamoux, F. 1953. Cyrène sous la monarchie des Battiades, Paris.

Cherstich, L. 2006a. S4: la tomba di Klearchos di Cirene. In Fabbricotti, E. et Menozzi, O. (eds), Cirenaica: studi, scavi e scoperte. Parte 1: nuovi dati da città e territorio. Atti del X Convegno di Archeologia Cirenaica (Chieti, 24-26 Novembre 2003) (British Archaeological Reports, International Series, 1488): 103-120.
Di Valerio, E., Cherstich, I., Carinci, M., Siciliano, F., D’Addazio, G. and Cinalli, A. 2005. Votive niches in funerary architecture in Cyrenaica (Libya). In Birault, C., Green, J., Kaldelis, A. and Stellatou, A. (eds) SOMA 2003: Symposium on Mediterranean Archaeology (British Archaeological Reports, International Series, 1391): 53-58.

Fabbricotti, E. 1996. "Divinità funerarie da Barce et Tolemaide ", Studi Miscellanei, 29: 117-126.

Fabbricotti, E. 2002. "Problemi di scultura cirenaica » in Quaderni i Archeologia della Libia, 16: 49-54.

Ferri, S. 1929. Divinità Ignote. Nuovi documenti di arte e di culto funerario nelle colonie greche, Vallecchi.

Frigerio, C. 1997. "Un esempio di architettura ellenistica funeraria a Cirene. La tomba S 388. ». Libya Antica 3: 51-73.

Frontisi-Ducroux, F. 2008. Les figures funéraires de Cyrène: stratégies de figuration de l'invisible ». In Image et religion dans l'Antiquité gréco-romaine, actes du colloque à Rome, 11-13 décembre 2003, Naples: 56 s.

Giovannini, V. 2014. Le sculture dal "Tempio di Cibele" nel santuario dei Dioscuri. In Luni, M. (ed.), Cirene "Atene d'Africa", VIII. La scoperta di Cirene, un secolo di scavi (1913-2013) (Monografie d'Archeologia Libica, 37), Rome: 67-89.

Llewellyn-Jones, L. 2003. Aphrodite's Tortoise, the Veiled Woman of Ancient Greece. Swansea.

Marini, S. 2012. Les bustes funéraires en Cyrénaïque à l'époque romaine: un exemple d'inluence de l'art funéraire égyptien sur celui des Cyrénéens? In Entre Afrique et Égypte: relations et échanges au sud de la Méditérranée à l'époque romaine.

Menozzi, O. 2015. Libya: Archaeology in War and archaeology of the war. In Skills and tools to the cultural heritage and cultural tourism management, vol. II, Teramo: 91-110.

Oldjira, M. and Walker, S. 2016. Stones of Memory, the presentation of individuals in the cemeteries of Cyrene. In Mugnai, N. et Nikolaus, J. De Africa Romaque: Merging Cultures accross North Africa, Leicester: 185-197.

Paribeni, E. 1959. Cyrene. Catalogo delle sculture di Cirene.

Rosenbaum, E. 1960. A Catalogue of Cyrenaican Portrait Sculpture, Londres, 1960.

Reynolds, J. et Thorn, J. C. 2005. "Cyrene's Thea figure discovered in the Necropolis ". Libyan Studies 36: 89-100.

Siciliano, F. 2006. Divinità funerarie Cirenaiche. Alcuni recenti rinvenimenti. In Fabbricotti, E. et Menozzi, O. (eds), Cirenaica: studi, scavi e scoperte. Parte 
1: nuovi dati da città e territorio. Atti del X Convegno di Archeologia Cirenaica (Chieti, 24-26 Novembre 2003) (British Archaeological Reports, International Series, 1488), Oxford: 409-422.

Thorn, J. C. 2005. The Necropolis of Cyrene. Two hundred years of exploration, Rome, 2005.
Walker, S. 1997. Mummy portraits in their Roman context. In Bierbrier, M.L. (ed.), Portraits and Masks: Burial Customs in Roman Egypt, London: British Museum Press.

Zagdoun, Mary-Anne. 2004. Les sculptures de la Libye antique au Musée du Louvre. Journal des Savants 1.1: 61-128. 